\title{
Shape coexistence and disappearance of pairing correlations in ${ }^{82} \mathrm{Sr}$
}

\author{
C. Baktash ${ }^{\text {a }}$, G. Garcia-Bermudez ${ }^{\text {a,b, }, ~}$, D.G. Sarantites ${ }^{c}$, W. Nazarewicz ${ }^{\text {d.e }}$, V. Abenante ${ }^{c}$, \\ J.R. Beene ${ }^{\text {a }}$, H.C. Griffin ${ }^{\text {f }}$, M.L. Halbert ${ }^{\text {a }}$, D.C. Hensley ${ }^{\text {a }}$, N.R. Johnson ${ }^{\text {a }}$, I.Y. Lee ${ }^{\text {a }}$, \\ F.K. McGowan ${ }^{a}$, M.A. Riley ${ }^{a, b, e}$, D.W. Stracener ${ }^{c}$, T.M. Semkow ${ }^{c, 2}$ and A. Virtanen ${ }^{a, b, 3}$ \\ a Physics Division, Oak Ridge National Laboratory, Oak Ridge, TN 37831, USA \\ - Joint Institute for Heavy-Ion Research, Oak Ridge, TN 37831, USA \\ c Department of Chemistry, Washington University, Saint Louis, MO 63130, USA \\ a Institute of Physics, Warsaw University of Technology, PL-00-662 Warsaw, Poland \\ - Oliver Lodge Laboratory, University of Liverpool, Liverpool L693BZ, UK \\ f Department of Chemistry, University of Michigan, Ann Arbor, MI 48109, USA
}

Received 18 July 1990

\begin{abstract}
Extensive high-spin band structures in ${ }^{82} \mathrm{Sr}$ have been established using proton- $\gamma-\gamma$ coincidence techniques. On the basis of the Woods-Saxon cranking calculations with pairing, four of these bands are interpreted to have prolate, oblate, or triaxial shapes. Pairing correlations are predicted to be very weak at high spins in this nucleus, and calculations with no static pairing successfully reproduce the experimentally observed crossing frequencies and alignments despite the fact that none of the bands displays a rigid-rotor behavior. It is concluded that observation of rigid-rotor behavior is neither necessary nor sufficient for the disappearance of static pairing in nuclei.
\end{abstract}

A rich variety of shape changes that include transition from noncollective oblate to highly collective superprolate [1], or the opposite, a change from prolate to oblate shapes $[2,3]$ has been reported in several of the transitional rare-earth nuclei. Similarly, rigid-rotor behavior and the presence of real band crossings have been observed and attributed to possible loss of pairing correlations at high spins in several medium-mass [4-6] and rare-earth [7-9] nuclei. The majority of these studies have focused on the rare-earth nuclei which are readily populated to very high spins. In comparison, the transitional nuclei near mass $A=80$ have received less attention, despite the fact that they are expected to manifest a similarly right pattern of shape and pairing varia-

1 Permanent address: Comision Nacional de Energia Atomica, 1429 Buenos Aires, Argentina.

2 Present address: Wadsworth Center for Laboratories and Research, New York State Department of Health, Albany, NY 12201, USA.

3 Present address: University of Jyväskylä, SF-40100 Jyväskylä, Finland. tions that are induced by rotation [10]. This is partly due to the experimental difficulty in studying very high spin states in this region, and partly because of the challenging task of the theoretical interpretation of the results.

In this paper we report experimental results and theoretical interpretation for four high-spin band structures in the transitional nucleus ${ }^{82} \mathrm{Sr}$. Because of its very soft core and the presence of large shell gaps at $Z=38$ and $N=44$ particle numbers, the ${ }^{82} \mathrm{Sr}$ nucleus is expected to be one of the best candidates in the region for the study of shape changes and quenching of the nuclear superfluidity. The high-spin states in ${ }^{82} \mathrm{Sr}$ were populated with the fusion-evaporation reaction ${ }^{52} \mathrm{Cr}\left({ }^{34} \mathrm{~S}, 2 \mathrm{p} 2 \mathrm{n}\right)$ by use of a $130 \mathrm{MeV}{ }^{34} \mathrm{~S}$ beam from the HHIRF facility at ORNL. The target was a stack of two self-supporting $275 \mu \mathrm{g} / \mathrm{cm}^{2}$ thick foils enriched in ${ }^{52} \mathrm{Cr}$. The experimental setup consisted of the ORNL Compton-suppression Spectrometer System with $18 \mathrm{Ge}$ detectors for discrete gammaray spectroscopy, 52 elements of the Spin Spectrometer [11] which recorded the associated gamma-ray multiplicity, and the Washington University Dwarf 
Ball [12] \#1. The Dwarf Ball is a $4 \pi$, 72-element CsI detector that affords light charged-particle identification with good energy resolution. This combination of experimental systems provided for both highresolution gamma-ray spectroscopy and complete exit channel selection. Demanding the coincidence triggering of at least one charged-particle and two or more Compton-suppressed $\mathrm{Ge}$ detectors, we recorded nearly 200 million events.

From these data we constructed a $3000 \times 3000$ channel $\gamma-\gamma$ coincidence matrix subject to the requirements that signals from at least ten $\mathrm{NaI}$ elements of the Spin Spectrometer, and one or two pro-

\#1 A Cs ( Tl) version of the device is described in ref. [13]. tons were present in each event. The resulting matrix, which was dominated by ${ }^{82} \mathrm{Sr}$, was used to construct the level scheme shown in fig. 1. Except for a few transitions, this level scheme is in agreement with that reported for lower spins by Haskins et al. [14]. Two new bands were discovered and four of the previously known bands have now been extended to spins that range from $20 \hbar$ to $27 \hbar$. The spin-parity assignments for states below $\sim 5 \mathrm{MeV}$ excitation are based on the angular distribution and polarization work of Haskins et al. (ref. [14]). Directional correlation of oriented nuclei (DCO) data from the present work were used to assign stretched E2 character to the gamma rays deexciting the higher-lying members of these bands. Because the $1005 \mathrm{keV}$ line is a triplet,

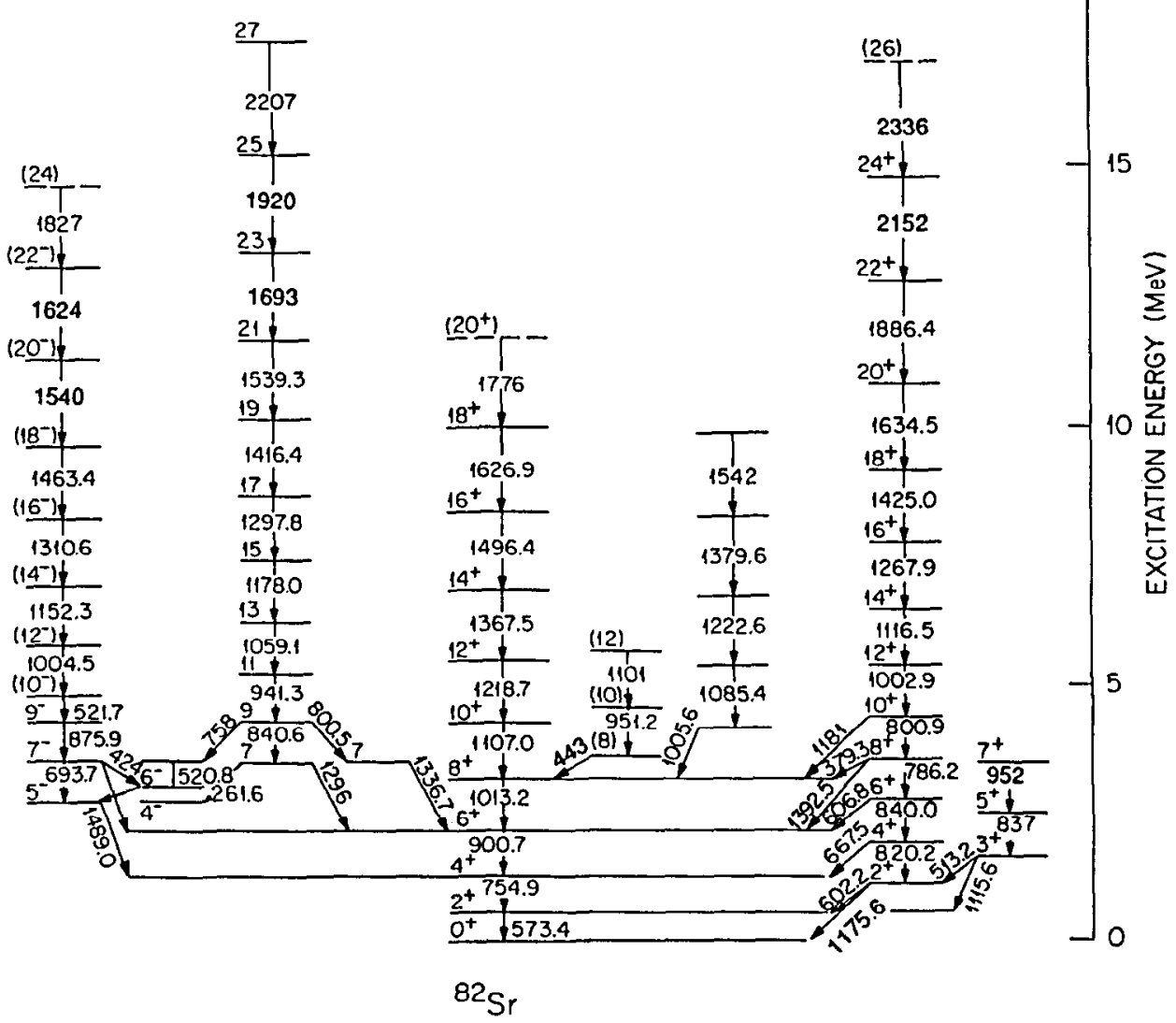

Fig. 1. A partial level scheme for ${ }^{82} \mathrm{Sr}$. Band labels are denoted by numbers in parentheses. 
spin-parity assignments of band 6 remain undetermined. Similarly, the parity of band 3 was not determined, although negative parity is favored on theoretical grounds as discussed below.

A theoretical analysis of these band structures in ${ }^{82} \mathrm{Sr}$ was carried out using the cranking approximation and the Strutinsky method that incorporates the Woods-Saxon potential as described in refs. $[15,16]$. The equilibrium deformation at a fixed value of $\omega$ was determined by minimizing the total routhian surface with pairing at each $\left(\beta_{2}, \gamma\right)$ grid point with respect to $\beta_{4}$. Details of the calculational procedure and technique are given in refs. $[17,18]$, where additional references may be found. In the following paragraphs we shall present a description of the band structures in ${ }^{82} \mathrm{Sr}$ which, according to these calculations, exhibit prolate, oblate, and triaxial shapes.

Prolate ground-state band (band 4 ): While the absolute minimum in the potential energy surface for the ground state corresponds to a spherical shape, collective rotation of a prolate shape quickly becomes favorable. As seen in fig. 2 , the deformation of the ground-state band (GSB) increases from spheri-

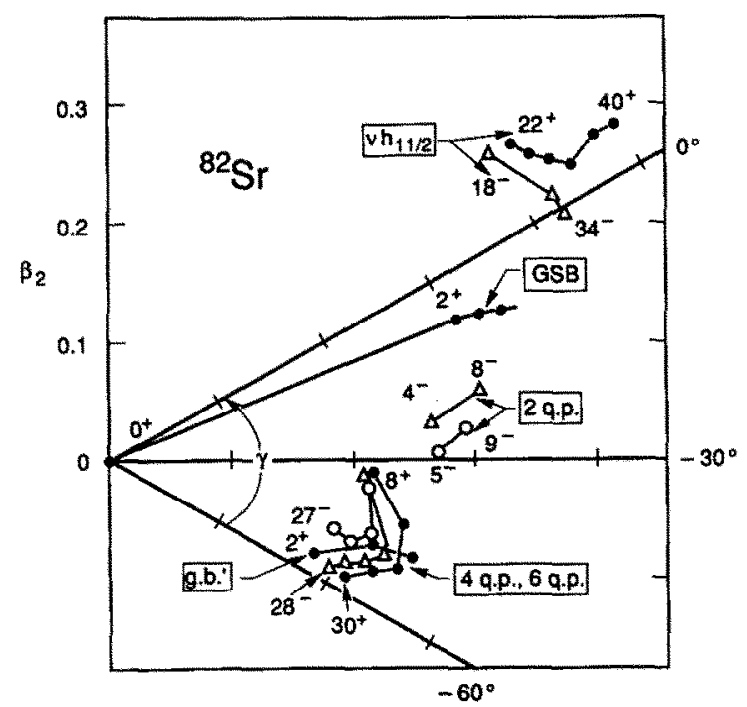

Fig. 2. Calculated equilibrium deformations in the $\left(\beta_{2}, y\right)$ plane for members of various multi-quasiparticle (q.p.) bands in ${ }^{82} \mathrm{Sr}$ : (i) GSB denotes the ground state band 4; (ii) 2q.p. refers to the low-spin members of bands 1 and 2 ; (iii) g.b.' is the quasigamma band and refers to the low-spin states in band 7; and (iv) 4q.p. refers to the high-spin states in bands 3 and 7 . The superdeformed configurations require excitation of the $\nu \mathrm{h}_{11 / 2}$ orbital. cal at spin zero, to a large value of $\beta_{2}=0.34$ at spin $I=6 \hbar$. Quasiparticle routhians calculated at this deformation show the $\mathrm{g}_{9 / 2}$ proton alignment to take place at a critical frequency of $\hbar \omega=0.56 \mathrm{MeV}$. A gradual $\mathrm{g}_{9 / 2}$ neutron crossing is delayed until $h \omega$ $=0.75 \mathrm{MeV}$. These predicted crossing frequencies are in agreement with the experimental data. Fig. 3 shows plots of the dynamic moments of inertia, $J^{(2)}$, as functions of the rotational frequency for the GSB in ${ }^{80} \mathrm{Sr}$ (ref. [19]) and ${ }^{82} \mathrm{Sr}$ nuclei. Both nuclei show first a gentle upbending at $\hbar \omega=0.55 \mathrm{MeV}$, followed by a second crossing at $\hbar \omega=0.75 \mathrm{MeV}$ which give rise to the corresponding peaks in the dynamic moments of inertia. (The deformation and pairing selfconsistent calculations for the GSB in ${ }^{80} \mathrm{Sr}$ are discussed in ref. [15].)

Oblate positive-parity band (band 7). In our calculations there exists a nearly oblate excited band which lies about $0.5 \mathrm{MeV}$ above the GSB, and may be identified at low spins with the experimental quasigamma band, namely the states below spin $I=7$ in band 7 . This quasigamma band strongly links via a $786.2 \mathrm{keV}$ transition to the aligned $(+, 0)$ band that is built on the $8_{2}^{+}$state. $[(\pi, 0)$ and $(\pi, 1)$ designate even-spin and odd-spin bands of parity $\pi$, respectively.] As discussed in ref. [20], the $\mathrm{g}_{9 / 2}$ quasiparticles exert a strongly polarizing force with the result

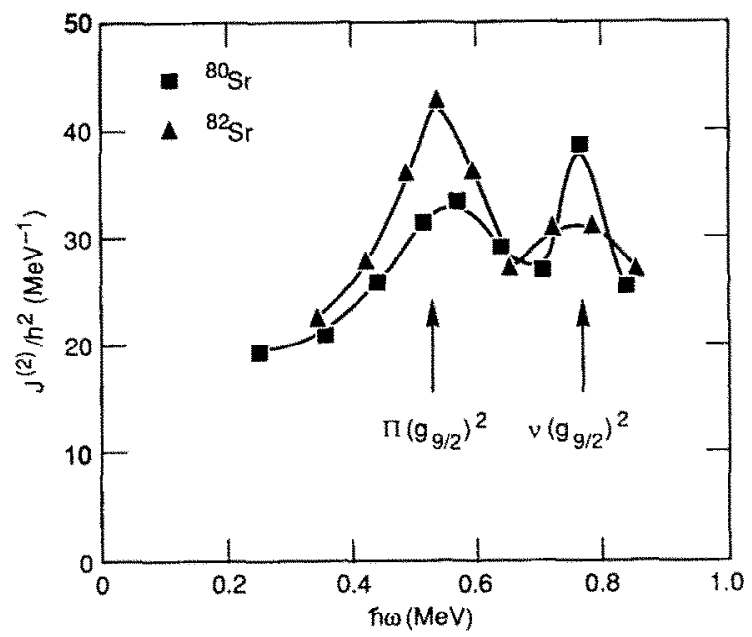

Fig. 3. Evolution of $J^{(2)}$ moments of inertia as functions of rotational frequency for the prolate ground-state bands in ${ }^{80} \mathrm{Sr}$ (squares), and ${ }^{82} \mathrm{Sr}$ (triangles). Arrows indicate the crossing frequencies of the first proton, and neutron $\mathrm{g}_{9 / 2}$ orbitals. 
that the positive-parity yrast sequence experiences a rapid shape change above $I=8 \hbar$. That is, excitation of the proton $\left(\mathrm{g}_{9 / 2}^{2}\right)$ orbitals triggers a shape change toward $\gamma=-30^{\circ}$, which is shortly followed by alignment of a $\mathbf{g}_{9 / 2}$ neutron pair. This results in a fourquasiparticle configuration for spins $I>14 \hbar$ ( $\hbar \omega>0.6$ $\mathrm{MeV})$ which has oblate shape $\left(\beta_{2}=0.22, \gamma=-50^{\circ}\right)$ and forms the $(+, 0)$ yrast sequence. This explanation is consistent with the crossing frequencies of the proton and neutron $g_{9 / 2}$ orbitals in the neighboring odd- $N\left(\hbar \omega \simeq 0.5 \mathrm{MeV}\right.$ in $\left.{ }^{81} \mathrm{Sr},{ }^{83} \mathrm{Zr}\right)$ and odd- $Z$ ( $\hbar \omega \simeq 0.52 \mathrm{MeV}$ in ${ }^{81} \mathrm{Rb},{ }^{83} \mathrm{Y}$ ) nuclei, respectively. Moreover, this interpretation of band 7 provides a natural explanation for the recently reported $g$-factors [21] of $0.7 \pm 0.1$ for the two lowest-lying $8^{+}$ states, which are otherwise difficult to understand. These experimental $g$-factors are different from the collective value of $g_{R}=0.4$, and also those expected for pure two-quasiparticle configurations $\left[g\left(\pi \mathrm{g}_{9 / 2}^{2}\right)\right.$ $\left.=+1.37, g\left(\nu \mathrm{g}_{9 / 2}^{2}\right)=-0.243\right]$. Assuming a simple cranking-model formula for the magnetic dipole moment [22] and using the experimentally deduced alignments of the $8_{1}^{+}$and $8_{2}^{+}$bands, we obtain values of $g\left(8_{1}^{+}\right)=0.67$, and $g\left(8_{2}^{+}\right)=0.72$. These $g$ factors are in good accord with the experimental values of $0.7 \pm 0.1$ reported for these states.

The next two $g_{9 / 2}$ proton orbitals are predicted to cross the Fermi level at frequencies of $\hbar \omega>1.1 \mathrm{MeV}$. The increase in $J^{(2)}$ at $\hbar \omega>1.0 \mathrm{MeV}$ in fig. $4 \mathrm{a}$ is associated with this transition to a six-particle configuration.

Triaxial and oblate negative-parity bands (bands 1 and 3 ). The negative-parity bands of both signatures are predicted to consist of two-quasiproton configurations, in agreement with the experimental systematics [23]. The calculated low-lying negative-parity configurations consist of particle-hole excitations from a negative-parity orbital to the $\mathrm{g}_{9 / 2}$ orbitals. The resulting equilibrium deformations of these bands correspond to triaxial shapes with $\beta_{2}=0.28$ and $-30^{\circ}<\gamma<-20^{\circ}$. Around $\hbar \omega=0.5 \mathrm{MeV}, \mathrm{g}_{9 / 2}$ neutron pair becomes aligned and drives the nucleus toward nearly oblate shape (see the four-quasiparticle states in fig. 2). It is predicted that the next proton quasiparticle alignment takes place around $\hbar \omega=0.75$ $\mathrm{MeV}$ for the $(-, 1)$ band, and has a large band interaction in agreement with the experimental data (fig. $4 b)$.

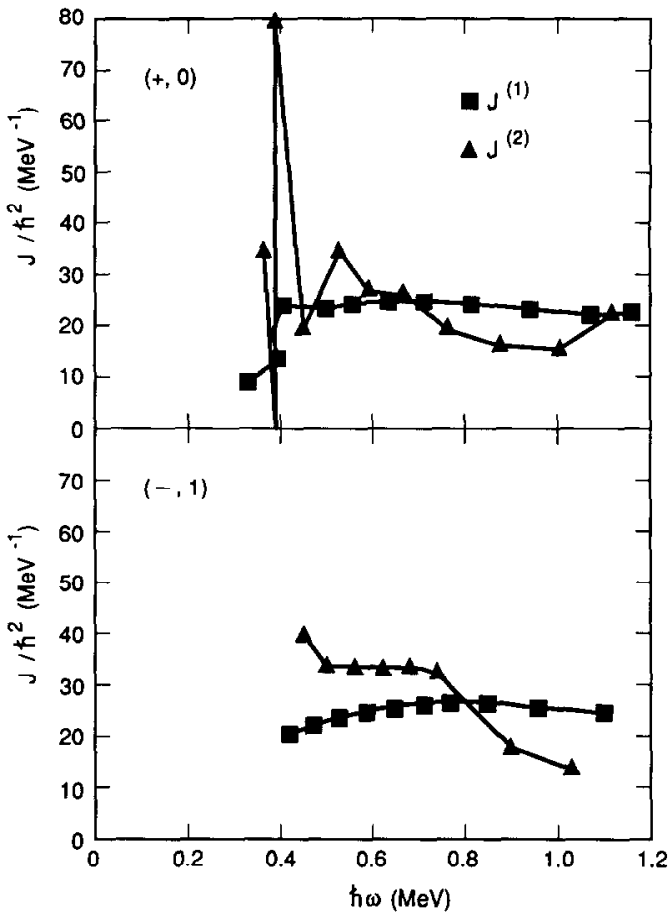

Fig. 4. Evolution of the $J^{(1)}$ (squares), and $J^{(2)}$ (triangles) moments of inertia as functions of rotational frequency for the (a) positive-parity $(+, 0)$, and (b) assumed negative-parity $(-, 1)$ bands in ${ }^{82} \mathrm{Sr}$.

Strongly-deformed prolate band. At spins $I>32 \hbar$ a large prolate deformation $\left(\beta_{2}>0.42\right)$ is predicted to set in as the lowest neutron $\mathrm{h}_{11 / 2}$ orbital becomes occupied (see fig. 2). The experimental $E_{\gamma}-E_{\gamma}$ correlation map shows the weak ridge-valley structure with a width of $\Delta E_{\gamma}=150 \mathrm{keV}\left[J^{(2)} / \hbar^{2}=26.7 \mathrm{MeV}^{-1}\right]$, that corresponds to $\beta_{2}=0.5$ for a deformed rigid rotor. However, we have not been able to identify any discrete band that could be associated with this ridgevalley structure.

Taken together, the above structures in ${ }^{82} \mathrm{Sr}$ reveal one of the richest patterns of shape coexistence observed so far in this mass region. To confirm our interpretation of these bands, lifetime measurements of the high-spin states are called for.

Several papers in the past few years have reported the onset of rigid-rotor behavior $\left(J^{(1)} \simeq J^{(2)} \simeq J_{\text {rigid }}\right)$ at high spins near $A \approx 80$ (e.g., ${ }^{84} \mathrm{Zr},{ }^{76,78} \mathrm{Kr},{ }^{72} \mathrm{Se}$ in refs. [4], refs. [5,6] and ref. [24], respectively) and $A \approx 170$ (e.g., ${ }^{168} \mathrm{Hf},{ }^{165} \mathrm{Yb}$ in ref. [7] and ref. [8]). It has been suggested by these authors that loss of 
static pairing correlations may be responsible for this behavior in these nuclei. In the present work we have investigated the question of static pairing correlations and its evolution with spin in ${ }^{82} \mathrm{Sr}$, which is an isotone of ${ }^{84} \mathrm{Zr}$.

At moderate to high spins, the equilibrium deformations obtained in the present analysis closely resemble those obtained from calculations without pairing correlations which are discussed in ref. [15]. Schematically, this is due to the presence of large shell gaps at $Z=38$ and $N=44$ particle numbers which remain practically constant up to very high rotational frequencies. As a result, proton, and to a lesser extent, neutron pairing are considerably reduced. For example, in a calculation that employs particle-number projection before variation, the proton pairing energy was estimated to be approximately $-1.5 \mathrm{MeV}$ at zero frequency. This is only 40 percent of the typical value of $-4 \mathrm{MeV}$. (Pairing encrgy is defincd as the difference between the total energy when calculated with and without pairing.) This reduction is even more dramatic for the lowest two-quasiproton configurations, where the proton pairing energy is reduced to only $-0.5 \mathrm{MeV}$. At yet higher spins, Coriolis antipairing essentially eliminates the remaining pairing correlation. Therefore, high-spin bands that involve aligned $\mathrm{g}_{9 / 2}$ particles, such as the $\mathrm{S}$-band are adequately described in the unpaired representation [15].

Further evidence in support of weakened static pairing correlations at high spins in ${ }^{82} \mathrm{Sr}$ comes from the fact that, experimentally, the $(-, 1)$ band crosses the $(+, 0)$ band to become the yrast band at a frequency of $\hbar \omega=0.88 \mathrm{MeV}$. In the present calculation, this can only be understood when pairing is neglected (see also ref. [9] for another good example in the rare-earth region).

It is worthwhile to note that, far from showing a rigid-rotor behavior, the $J^{(2)}$ moment of inertia of the negative-parity band in fig. $4 \mathrm{~b}$ gradually decreases at frequencies above $\hbar \omega=0.7 \mathrm{MeV}$. The fact that highspin bands in ${ }^{82} \mathrm{Sr}$ show no rigid-rotor behavior, even if their properties are well described in calculations without pairing, is a clear indication that loss of static pairing need not necessarily be accompanied by a rigid-rotor behavior. Conversely, since in addition to pairing correlations the moments of inertia reflect, and are sensitive to, other properties of the band (e.g., shape parameters and alignments), their behavior cannot be indicative of the evolution of the pairing strength alone. Thus, inferences regarding the superfluid-to-normal phase transition should not be based solely on the behavior of the moments of inertia. (See also ref. [25].)

In summary, we have identified several collective bands in ${ }^{82} \mathrm{Sr}$ that exhibit coexistence of prolate, triaxial, and oblate shapes. In addition, a weak ridgevalley structure in the $\gamma-\gamma$ correlation map hints at the presence of prolate superdeformed structures. Theoretical calculations with no pairing successfully reproduce the experimentally observed crossing frequencies and alignments of these bands at high spins. Nevertheless, no rigid-rotor behavior was observed in any of these bands. It is concluded that observation of a rigid-rotor behavior is neither necessary nor sufficient to indicate the absence of static pairing in nuclci.

Oak Ridge National Laboratory is operated by Martin Marietta Energy systems, Inc. for the US Department of Energy under contract No. DE-AC05$840 \mathrm{R} 21400$. This work was supported in part by the US Department of Energy under grant No. DE-FG0288ER-40406, NSF-CONICET US-Latin American Cooperative Science Program, and the Polish ministry for National Education under grant No. CPBP01.09.

\section{References}

[1] P.J. Twin et al., Phys. Rev. Lett. 57 (1986) 811.

[2] A. Pakkanen et al., Phys. Rev. Lett. 48 (1982) 1530.

[3] C. Baktash et al., Phys. Rev. Lett. 54 (1985) 978.

[4] H.G. Price ct al., Phys. Rev. Lett. 51 (1983) 1842.

[5] M.S. Kaplan et al., Phys. Lett. B 215 (1988) 251.

[6] C.J. Gross et al., Nucl. Phys. A 501 (1989) 367.

[7] K. Chapman et al., Phys. Rev. Lett. 51 (1983) 2265

[8] C. Schuck et al., Phys. Lett. B 142 (1984) 253.

[9] M.A. Riley et al., Phys. Rev. Lett. 60 (1988) 553; J. Phys. G 16 (1990) L67.

[10] W. Nazarewicz and T. Werner, in: Nuclear structure of the zirconium region, eds. J. Eberth et al. (Springer, Berlin, $1988)$ p. 277

[11] M. Jääskeläinen et al., Nucl. Instrum. Methods 204 (1983) 385.

[12] D.W. Stracener et al., Nucl. Instrum. Methods 294 (1990) 485. 
[13] D.G. Sarantites et al., Nucl. Instrum. Methods A 264 (1987) 319.

[14] P.S. Haskins et al., Phys. Rev. C 32 (1985) 1897.

[15] W. Nazarewicz et al., Nucl. Phys. A 435 (1985) 397.

[16] J. Dudek et al., Phys. Rev. C 35 (1987) 1489.

[17] U.J. Hüttmeier et al., Phys. Rev. C 37 (1988) 118.

[18] E.F. Moore et al., Phys. Rev. C 38 (1988) 696.

[19] R. Davie et al., Nucl. Phys. A 463 (1987) 683.

[20] R. Bentsson and W. Nazarewicz, in: Proc. XIX Winter School (Zakopane, Poland, 1984), ed. Z. Stachura, p. 171.
[21 ] A.I. Kucharaska et al., J. Phys. G 15 (1989) 1039.

[22] S. Frauendorf, Phys. Lett. B 100 (1981) 219.

[23] L. Funke et al., Nucl. Phys. A 355 (1981) 228.

[24] J.H. Hamilton, in Proc. Workshop on Microscopic models in nuclear structure physics, eds. M.W. Guidry et al. (World Scientific, Singapore, 1989) p. 45.

[25] J.D. Garrett, in Proc. Intern. Symp. on Heavy ion physics, J. Phys. Soc. Jap. 54, Suppl. II (1985) 456. 